\title{
Comparison of Sleep Quality in Pre-HAART and on HAART HIV Patients
}

\author{
Felisitas Farica Sutantoyo ${ }^{1}$, Paulus Sugianto ${ }^{1}$, Wardah Rahmatul Islamiyah ${ }^{1}$ \\ ${ }^{1}$ Department of Neurology, Faculty of Medicine Airlangga University, \\ Dr. Soetomo General Hospital, Surabaya, Indonesia \\ Corresponding Author: Paulus Sugianto (paulus.sugianto.unair@gmail.com)
}

\begin{abstract}
Background/aim: People with HIV are often reported to experience poor sleep quality. Factors that can cause poor sleep quality in HIV patients, including the use of ARV therapy. The pathophysiology of poor sleep quality in HIV patients is still unclear. Poor sleep quality is one of the complaints in the late stages of HIV infection, where it is suspected that HIV itself affects the biological center of sleep. This study was aimed to determine the comparison of sleep quality in HIV patients pre-HAART and with HAART more than 1 year.
\end{abstract}

Method: This study uses data collection methods in the form of interviews with samples which will later be presented in the form of analytical observational study. Consecutive data collection on HIV patients before and after ARV therapy who was outpatient at the VCT outpatient clinic Dr. Soetomo General Hospital from June-July 2021. Data analysis using SPSS Version 23 for windows

Results: A total of 40 subjects were divided into HIV pre-HAART and on HAART. From the statistical analysis using SPSS program significant with $\mathrm{p}=0.004$ (OR 15.5 (95\% CI (1.73-139.6))

Conclusion: HIV patients on HAART for more than 1 year are known to have increased 15.5 times the risk of poor sleep quality

Keywords: HIV, HAART, sleep quality

\section{INTRODUCTION}

People with HIV are often reported to experience poor sleep quality.[1] Factors that can cause poor sleep quality in HIV patients, including the use of ARV therapy.[2] According to data from the World Health Organization (WHO) globally, it is estimated that the presentation of HIV sufferers in 2020 was estimated at 35.3 million people and 9.7 million of them had received ARV therapy. In Indonesia, according to data from the Central Statistics Agency (BPS) up to June 2021, the number of people living with HIV was around 108,600. The cumulative number according to the highest risk factors is heterosexual, homosexual, and the use of Intravenous Drug User (IVDU) with a total of 26,158 patients. Based on the age group the most ranged from the age of 20-29 years as many as 15,305 patients. The highest number was in DKI Jakarta with 24,807 patients, in East Java with 14,285 patients, and in Papua with 11,871 patients.[3]

Poor sleep quality in HIV sufferers is caused by conditions related to HIV itself and its systemic complications.[4] Poor sleep quality is often found in patients with advanced HIV disease due to the use of ARVs. A number of studies have reported factors that can lead to poor sleep quality in HIV patients. Initial investigations analyzing sleep architecture using polysomnography, focused on biological relationships, particularly the manifestations of HIV infection in the Central Nervous System (CNS) and changes in immune response.[5] In evaluation the use of polysomnography is not indicated in paradoxical insomnia or poor sleep quality sleep apnea.[6] 
Factors that can cause poor sleep quality in HIV patients include the use of ARV therapy, an antiretroviral consisting of NRTI and NNRTI groups. The NRTI group consists of zidovudine (ZDV or AZT), lamivudine (3TC), stavudine (d4T), abacavir (ABC), tenofovir disoproxil fumarate (TDF), and emtricitabine (FTC). The NNRTI class includes nevirapine, delavirdine, and efavirenz. Didanosine and efavirenz can cause nightmares and depression.[2] EEG images in HIV patients taking efavirenz show a decrease in sleep efficiency with difficulty in starting sleep, an increase in the number of wakes during sleep, and a decrease in the duration of REM sleep.[7,8]

The most optimum time to start ARV therapy is CD4 cells between 200$350 / \mathrm{mm} 3$. ARV is recommended in patients with pulmonary TB with severe bacterial infection and CD4 $<350 / \mathrm{mm} 3$, and in pregnant women with clinical stage CD4 < $350 / \mathrm{mm} 3$. Several studies have shown poor sleep quality in HIV patients who have a CD4 count $<200$ cells/mm3.[6,9]

Sleep quality was subjectively assessed based on clinical interviews with patients and their sleep partners. Completing a complete sleep diary/log is also useful for evaluating insomnia. Physical and laboratory examinations are also indicated. Polysomnography is a measurement of brain waves during sleep to determine the stages of sleep so that objective data is obtained about the stages and architecture of disturbed sleep.[10] Epidemiological studies of poor sleep quality generally use selfreport measures to evaluate insomnia. The widespread use of the Pittsburgh Sleep Quality Index (PSQI) in various populations, allows for multiple comparison studies. The PSQI consists of 19 self-rated questions and 5 questions rated by a sleep partner or roommate (only self-rated questions are used in the rating scale). The PSQI contains 15 multiple-choice questions that ask about the frequency of sleep disturbances and subjective sleep quality and 4 questions that ask about typical bedtime, wake time, sleep latency, and sleep duration. All questions are short and easy to understand and have been adapted so that they can be used by doctors or research assistants. PSQI produces seven values that correspond to the existing domain. Each score component ranges from 0 (no difficulty) to 3 (severe difficulty). The component values are summed to produce a global score (range 0-21). A global PSQI score $>5$ is considered a significant sleep disturbance. Cutoff values are not available for each component.[2,9] Based on this description, a study is proposed on the comparison of sleep quality in HIV patients PRE-HAART with patient on HAART

\section{METHOD}

This study uses data collection methods in the form of interviews with samples which will later be presented in the form of analytical observational study. Consecutive data collection on HIV patients before and after ARV therapy who was outpatient at the VCT outpatient clinic Dr. Soetomo General Hospital from June-July 2021. To assess sleep quality, the PSQI score which has been translated into Indonesian was used.

The questionnaire used to assess sleep quality with the PSQI consisted of 19 self-rate questions containing 15 multiple choices asking about the frequency of subjective poor sleep quality with 4 questions about sleep initiation time, wake time, latency and sleep duration. Each score component ranges from 0 (no difficulty) to 3 (severe difficulty). The component values are summed to produce a global score (range 0-21). A global PSQI score $>5$ is considered a significant poor sleep quality [9]

The inclusion criteria established in this study were: 1) HIV positive patients, 2) Patients aged 18 years, 3) Patients who had not received ARV therapy and who had been on ARV treatment for at least 1 year of routine ARV use 4) Cooperative patients and willing to be included in the study by signing a letter of consent after an 
explanation (informed consent). The Patients with aphasia (sensory or motor). exclusion criteria for this study were:1) Patients with decreased consciousness, 2) Data analysis using SPSS Version 23 for windows

\section{RESULT}

Table 1: Characteristics of Subjects

\begin{tabular}{|c|c|c|c|}
\hline \multirow{2}{*}{\multicolumn{2}{|c|}{ Variable }} & Pre-HAART & On HAART \\
\hline & & $\mathbf{n}(\%)$ & $\mathbf{n}(\%)$ \\
\hline \multicolumn{2}{|c|}{ Age (years) mean (min-max) } & $36.4(24-59)$ & $38.2(37-59)$ \\
\hline \multirow[t]{2}{*}{ Age (years) } & $\leq 30$ & $7(17.5)$ & $3(7.5)$ \\
\hline & $>30$ & $13(32.5)$ & $17(42.5)$ \\
\hline \multirow[t]{2}{*}{ Gender } & man & $12(30)$ & $13(32.5)$ \\
\hline & female & $8(20)$ & $7(17.5)$ \\
\hline \multirow[t]{2}{*}{ Marital status } & Married & $11(27.5)$ & $9(22.5)$ \\
\hline & Not married & $9(22.5)$ & $11(27.5)$ \\
\hline \multirow[t]{2}{*}{ Level of education } & Senior High School & $12(30)$ & $8(20)$ \\
\hline & Academy/ collage & $8(20)$ & $12(30)$ \\
\hline \multirow[t]{2}{*}{ ARV regimen } & TDF+3TC(FTC)+NVP & - & $6(15)$ \\
\hline & $\mathrm{TDF}+3 \mathrm{TC}(\mathrm{FTC})+\mathrm{EFV}$ & - & $14(35)$ \\
\hline \multirow[t]{4}{*}{ Job Status } & Government Employers & $6(15)$ & $4(10)$ \\
\hline & Private employer & $5(12.5)$ & $8(20)$ \\
\hline & Entrepreneur & $6(15)$ & $7(17.5)$ \\
\hline & Does not work & $3(7.5)$ & $1(2.5)$ \\
\hline \multirow[t]{2}{*}{ Quality sleep } & Good & $9(22.5)$ & 0 \\
\hline & Poor & $11(27.5)$ & $20(50)$ \\
\hline \multirow[t]{2}{*}{ CD4 Count } & $\leq 200$ & $19(47.5)$ & $9(22.5)$ \\
\hline & $>200$ & $1(2.5)$ & $11(27.5)$ \\
\hline \multicolumn{2}{|c|}{ CD4 Count mean (min-max) } & $73.05(9-262)$ & $158.4(20-329)$ \\
\hline AZT: Zidovudine & \multicolumn{2}{|c|}{ FTC: emtricitabine or emtricitabine } & EFV: Efavirenz \\
\hline
\end{tabular}

The study involved 40 patients with HIV subjects who met the eligibility criteria, grouped into groups with ARV therapy (20 subjects) and without ARV (20 subjects).
The sample characteristics assessed in this study included gender, age, marital status, level of education, ARV regimen in sample with ARV therapy, Job status, and duration of using ARV at Table 1.

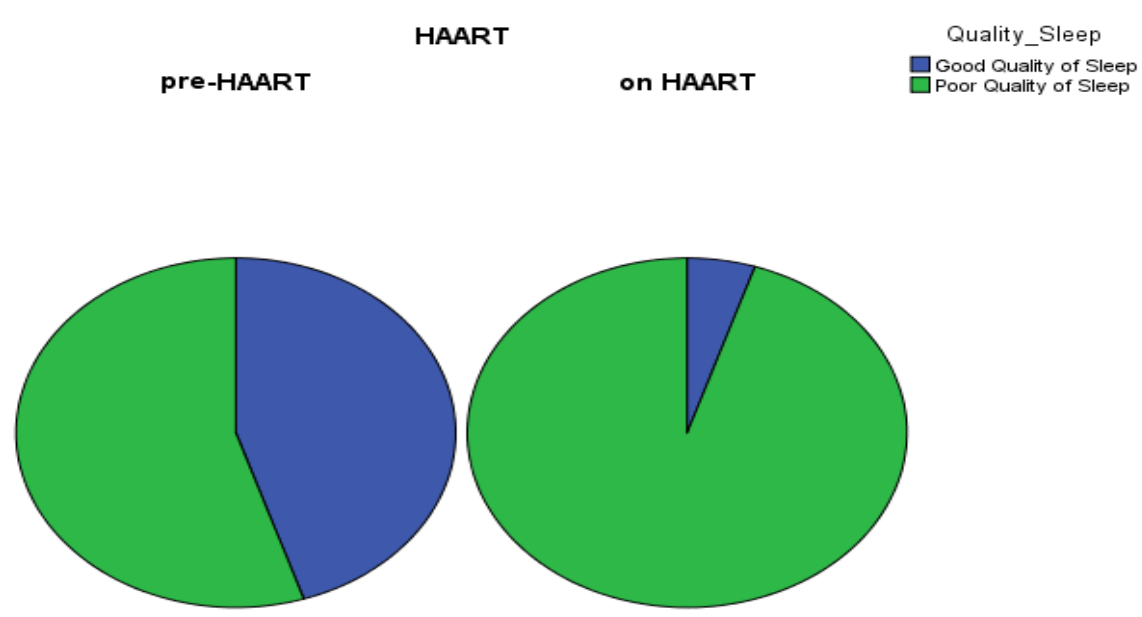

Figure 1: Comparison of sleep quality between pre-HAART and On-HAART HIV patients $\mathrm{p}=0.004($ OR $15.5(95 \%$ CI (1.73-139.6) $)$

The results of the comparison of sleep quality between HIV patients' preHAART and On HAART are shown in Figure 1. where the results with the Mann Whitney test are known to be $\mathrm{p}=0.004$ (OR 15.5 (95\% CI (1.73-139.6)) which means that there is a significant difference sleep quality with ARV administration, with the risk of increasing poor sleep quality as much as 15.5 times. In this study, a comparison test of sleep quality with the overall variables included; gender, age, 
marital status, level of education, ARV regimen, Job status, ARV use and CD 4 count in table 2, it is known that the results are statistically significant only ARV use with $\mathrm{p}=0.004$ as well as multivariate analysis to determine the factor of poor sleep quality and the results are only due to the use of ARVs (table 3).

Table 2: Comparison of sleep quality with all variables

\begin{tabular}{|c|c|c|c|c|}
\hline \multicolumn{2}{|c|}{ Variable } & \multirow{2}{*}{$\begin{array}{c}\text { Good Quality of Sleep } \\
\mathbf{n}(\%)\end{array}$} & \multirow{2}{*}{$\begin{array}{c}\begin{array}{c}\text { Poor Quality of Sleep } \\
\mathbf{n}(\%)\end{array} \\
3(7.5)\end{array}$} & \multirow{2}{*}{$\begin{array}{c}\mathbf{P *} \\
0.212\end{array}$} \\
\hline \multirow[t]{2}{*}{ Age (years) } & $\leq 30$ & & & \\
\hline & $>30$ & $1(2.5)$ & $16(40$ & \\
\hline \multirow[t]{2}{*}{ Gender } & Man & 0 & $13(32.5)$ & \multirow[t]{2}{*}{0.352} \\
\hline & Woman & $1(2.5)$ & $6(15)$ & \\
\hline \multirow[t]{2}{*}{ Marital status } & Married & 0 & $10(25)$ & \multirow[t]{2}{*}{0.149} \\
\hline & Not married & $1(2.5)$ & $9(22.5)$ & \\
\hline \multirow[t]{2}{*}{ Level of education } & Senior High School & 0 & $8(20)$ & \multirow[t]{2}{*}{0.582} \\
\hline & Academy/ collage & $1(2.5)$ & $11(27.5)$ & \\
\hline \multirow[t]{2}{*}{ Job Status } & Does not work & 0 & $1(2.5)$ & \multirow[t]{2}{*}{0.463} \\
\hline & Working & $1(2.5)$ & $18(45)$ & \\
\hline \multirow[t]{2}{*}{ Used ARV } & Pre-HAART & $1(2.5)$ & $19(47.5)$ & \multirow[t]{2}{*}{$\dagger 0.004$} \\
\hline & On HAART & $1(2.5)$ & $19(47.5)$ & \\
\hline \multirow[t]{2}{*}{ CD4 Count } & $\leq 200$ & $1(2.5)$ & $8(20)$ & \multirow[t]{2}{*}{0.116} \\
\hline & $>200$ & 0 & $11(27.5)$ & \\
\hline
\end{tabular}

Table 3: Multivariate analysis

Table 3: Multivariate analysis
\begin{tabular}{|l|c|c|c|}
\hline \multicolumn{1}{|c|}{ Variable } & Score & df & P* \\
\hline Age (years) & 1955 & 1 & 0.162 \\
\hline Gender & 0.186 & 1 & 0.666 \\
\hline Marital status & 0.861 & 1 & 0.353 \\
\hline Level of education & 0.702 & 1 & 0.402 \\
\hline ARV regimen & 2.456 & 1 & 0.117 \\
\hline Job Status & 1,271 & 1 & 0.260 \\
\hline Used ARV & 1.026 & 1 & $\dagger 0.004$ \\
\hline CD4 Count & 1,287 & 1 & 0.257 \\
\hline
\end{tabular} Multivariate logistic regression analysis
† Significant

\section{DISCUSSION}

Sleep has a function for homeostasis, energy conservation, brain restoration, increased immune function, and temperature regulation. The hypnotoxin theory explains that only in a state of sleep can the buildup of hypnotoxins in the body be detoxified. Adenosine, interleukins, tumor necrosing factors, prostaglandins, lipopolysaccharides and d (delta)-producing proteins play a role in mediating the homeostatic drive to sleep. During sleep, energy in the brain (ATP, glycogen, adenosine) will increase again because the use of energy and oxygen decreases by $15-20 \%$ compared to when awake. The restorative theory suggests that during sleep growth hormone is produced mainly during deep sleep.[11]

An abnormal sleep cycle is also known as poor sleep quality which is characterized by an imbalance between the Non Rapid Eye Movement (NREM) and Rapid Eye Movement (REM) phases. Types of poor sleep quality include: insomnia, hypersomnia, parasomnias, and disturbances in the sleep-wake cycle. Insomnia is defined as an inadequate perception of the quantity or quality of sleep. Insomnia is diagnosed through anamnesis in the form of difficulty initiating sleep, often waking from sleep, difficulty falling asleep again after waking up at night, quickly waking up in the morning which can cause fatigue, impaired concentration, and impaired memory.[12] Hypersomnia is a state of remaining drowsy even though the amount of sleep is adequate. Parasomnias describe unwanted states that occur while sleeping.[13]

Poor sleep quality often occurs early in HIV disease, before HIV symptoms appear and continues throughout the course of the disease.[1,4] In a study of HIV seropositive patients in outpatient clinics, about $73 \%$ complained of poor sleep quality.[14] Sleep problems in HIV patients which showed a prevalence ranging from $43 \%$ to $100 \%$. And evaluated $1,821 \mathrm{HIV}$ patients and found that $32 \%$ had poor sleep quality.[14]

The pathophysiology of poor sleep quality in HIV patients is still unclear. Poor sleep quality is one of the complaints in the late stages of HIV infection, where it is suspected that HIV itself affects the biological center of sleep. In HIV infection 
there are changes in the hypothalamicpituitary-adrenal axis in the form of loss of circadian rhythm and changes in endogenous substances that have an important role in sleep regulation such as neurotransmitters, opiate peptides, hormones and cytokines.[6]

In other study that 84 of $115(73 \%)$ outpatients with HIV experienced poor sleep quality. They need more than 1 hour to start sleeping and sleep duration is less than 2 hours, while good sleepers need less than 15 minutes to start sleeping and sleep duration is more than 2 hours $(\mathrm{p}<0.001)$.[2] Drug users in HIV patients experienced an increase in poor sleep quality up to $68 \%$, as well as a tendency to increase beta microglobulin levels.[9]

In this study, it was found that from the overall results of the data it was statistically significant that the use of ARVs $>1$ year affected sleep quality after being compared with other factors. A number of studies have reported the factors that contribute to the onset of poor sleep quality in people with HIV and AIDS. The initial investigations were primarily laboratorybased, analyzing sleep architecture using polysomnography and with a focus on biological relationships, particularly manifestations of central nervous system (CNS) HIV infection and altered immune responses. This emphasis has shifted to epidemiological studies to evaluate insomnia and factors associated with insomnia. As a direct effect of HIV infection on sleep, several studies have also considered the effect of other variables, including ARV drugs, mental illness, use of sleeping pills and alcohol use. The relationship between sleep quality and CD4 cell count remains unclear. Insomnia is a frequently reported adverse effect in association with antiretroviral therapy. However, studies evaluating ARVs and zidovudine as a group have not shown a significant effect. The exception is efavirenz, which has been evaluated and found to be an independent predictor of insomnia.[2,5]
Sample in Europe as poor sleepers with a score PSQI $>5$ as cutoff. Similarly, a prevalence of $30 \%$ was reported in 1800 Japanese adults. This study shows that insomnia is common in the general population. It is well recognized that there is a link between physical illness and insomnia, and this has been shown in several community studies. PSQI has also been used in samples of patients who are sick. In one study of patients with breast cancer, $61 \%$ were categorized as poor sleepers. A further study of 89 hemodialysis patients found that $63(71 \%)$ were poor sleepers. The question is whether the prevalence of insomnia in people with HIV is comparable to that in the general population or in people with other chronic diseases[15,16]

Although sleep disturbances are frequently reported in people with HIV and AIDS, only a few studies have systematically evaluated their prevalence. Only one study identified using the PSQI to classify as poor sleepers. In a crosssectional survey, found that 84 of $115(73 \%)$ outpatients with HIV infection had insomnia.[8] They needed more than 1 hour to sleep compared to less than 15 minutes in good sleepers $(p<0.001)$ and 2 hours less sleep $(p<0.001)$.[6] Two further studies reported mean global PSQI scores exceeding the cutoff for insomnia in the population with HIV, 9.0 (standard deviation 4.4) and 10.0 (standard deviation 5.0 ), but did not report the prevalence of poor sleepers. Insomnia alone was reported by two studies using direct questioning and they found prevalence of $29 \%$ and $56 \%$, respectively. None of these studies used controls, so it is necessary to consider the effect of confounding variables. [17]

The limitation of this study is the short duration of the study. This study used subjects in certain populations in certain places; hence, this study's results cannot describe the same conditions in different populations and places. Further research is needed to improve this study's results using a larger sample size involving various types 
of cancer and using cohort research methods.

\section{CONCLUSION}

This study showed a high proportion of poor sleep quality in HIV patients on HAART for more than a year with a 15.5 times increased risk compared to preHAART.

Competing Interests: No competing interests were disclosed.

Conflict of Interest: The authors declare no conflict of interest, financial or otherwise.

Acknowledgments: declared none.

Ethical Approval: This study has obtained ethical clearance issued by the Research Ethic Commission of Faculty of Medicine, Airlangga University, Soetomo General Hospital, Surabaya, East Java, Indonesia.

\section{REFERENCES}

1. Ren J, Zhao M, Liu B, Wu Q, Hao Y, Jiao $\mathrm{M}$, et al. Factors Associated With Sleep Quality in HIV. J Assoc Nurses AIDS Care. 2018;29(6):924-31. DOI: 10.1016/j.jana. 2018.04.006

2. Abdu Z, Dule A. Poor quality of sleep among HIV-positive persons in Ethiopia. HIV/AIDS-Res Palliat Care. 2020;12:621-8.

3. Mahamboro DB, Fauk NK, Ward PR, Merry MS, Siri TA, Mwanri L. HIV stigma and moral judgement: Qualitative exploration of the experiences of HIV stigma and discrimination among married men living with HIV in Yogyakarta. Int $\mathbf{J}$ Environ Res Public Health. 2020;17(2):115.

4. Gutierrez J, Tedaldi EM, Armon C, Patel V, Hart R, Buchacz K. Sleep disturbances in HIV-infected patients associated with depression and high risk of obstructive sleep apnea. SAGE Open Med. 2019;7: 205031211984226.

5. Spudich S, Peterson J, Fuchs D, Price RW, Gisslen M. Potential for early antiretroviral therapy to reduce central nervous system HIV-1 persistence. Aids. 2019;33 (December 2018):S135-44.

6. Faraut B, Malmartel A, Ghosn J, Duracinsky M, Leger D, Grabar S, et al.
Sleep Disturbance and Total Sleep Time in Persons Living with HIV: A CrossSectional Study. AIDS Behav. 2018;22 (9): 2877-87. DOI: $10.1007 / \mathrm{s} 10461-018-2179-1$

7. Meghdadi AH, Berka C, Richard C, Rupp G, Smith S, Stevanović Karić M, et al. EEG event related potentials in sustained, focused and divided attention tasks: Potential biomarkers for cognitive impairment in HIV patients. Clin Neurophysiol. 2021;132(2): 598-611.

8. Alikhani M, Ebrahimi A, Farnia V, Khazaie H, Radmehr F, Mohamadi E, et al. Effects of treatment of sleep disorders on sleep, psychological and cognitive functioning and biomarkers in individuals with HIV/AIDS and under methadone maintenance therapy. J Psychiatr Res. 2020;130:260-72. DOI: 10.1016/j.jpsychires.2020.07.043

9. Yan DQ, Huang YX, Chen X, Wang M, Li J, Luo D. Application of the Chinese Version of the Pittsburgh Sleep Quality Index in People Living With HIV: Preliminary Reliability and Validity. Front Psychiatry. 2021;12 (July):1-8.

10. Schwartz WJ, Klerman EB. Circadian Neurobiology and the Physiologic Regulation of Sleep and Wakefulness. Neurol Clin. 2019;37(3):475-86.

11. Wang F, Boros S. The effect of physical activity on sleep quality: a systematic review. Eur J Physiother. 2021;23(1):11-8. DOI: 10.1080/21679169.2019.1623314

12. Chokroverty Sudanshi. Sleep Disorders Medicine. Vol. 4, Springer. 2017. 740-748 p.

13. Byun JH, Kim KT, Moon H jin, Motamedi GK, Cho YW. The first night effect during polysomnography, and patients' estimates of sleep quality. Psychiatry Res. 2019;274 (February):27-9. DOI: 10.1016/ j.psychres. 2019.02.011

14. Saberi P, Comfort M, Sheon N, Johnson MO. Qualitative study of the quality of sleep in marginalized individuals living with HIV. Patient Prefer Adherence. 2013;7:499507.

15. Hakkers CS, Hermans AM, van Maarseveen EM, Teunissen CE, Verberk IMW, Arends JE, et al. High efavirenz levels but not neurofilament light plasma levels are associated with poor neurocognitive functioning in asymptomatic HIV patients. J Neurovirol. 2020;26(4):572-80. 
16. Celesia B. M., Sofia S. A., Rapisarda L., Maresca M., Vinci L., Gussio M., et al. Anxiety, depression and sleep disturbances in hiv+ patients chronically treated with an efavirenz-based regimen. Infect Dis Trop Med. 2017;3(2):e394.

17. Irwin MR, Olmstead R, Carroll JE. Sleep disturbance, sleep duration, and inflammation: A systematic review and meta-analysis of cohort studies and experimental sleep deprivation. Biol Psychiatry. 2016;80(1):40-52. DOI: 10.1016/j.biopsych.2015.05.014

How to cite this article: Sutantoyo FF, Sugianto $\mathrm{P}$, Islamiyah WR. Comparison of sleep quality in pre-HAART and on HAART HIV patients. International Journal of Research and Review. 2021; 8(10): 14-20. DOI: https://doi.org/ 10.52403/ijrr.20211003 